\title{
EL DESAPARECIDO "NUEVO PLANO PARA EL HOSPITAL REAL DE SAN LÁZARO”, UN POSIBLE PROYECTO DE SEBASTIÁN VAN DER BORCHT
}

\author{
THE MISSING "NEW FLOOR PLAN FOR THE ROYAL \\ HOSPITAL OF SAN LÁZARO”, A POSSIBLE PROJECT \\ BY SEBASTIÁN VAN DER BORCHT
}

\author{
Fernando Vilaplana Villajos \\ Universidad de Sevilla. España \\ ORCID: 0000-0001-5803-1764 \\ fvilaplana@us.es
}

\begin{abstract}
Este artículo expone la hipótesis de que el ingeniero militar Sebastián van der Borcht redactó un proyecto para el hospital de San Lázaro de Sevilla. Se muestra a continuación una evidencia epistolar de que existió este proyecto, y a través de los cambios formales que se produjeron en el edificio y la documentación escrita de principios del siglo XIX constatamos que esta transformación se produjo finalmente, aunque no podemos demostrar si las obras se ejecutaron bajo la dirección del propio Van der Borcht. Esta evolución de la leprosería, de origen medieval, hacia el modelo de hospital sanitario de carácter ilustrado nunca se había descrito y aporta nuevos valores a los ya conocidos en el hospital más antiguo, aun en funcionamiento, de España.

Palabras clave: Sebastián van der Borcht; arquitectura hospitalaria; lepra; ingenieros militares; Ilustración.
\end{abstract}

This paper presents the hypothesis that the military engineer Sebastián van der Borcht drafted a project for the San Lázaro Hospital in Seville. The following shows epistolary evidence that this project existed and we confirm that this transformation finally took place through the formal changes produced in the building and the written documentation of the early $19^{\text {th }}$ century, although we cannot prove whether the works were executed under the direction of Van der Borcht himself. This evolution of the leprosarium, of medieval origin, towards a healthcare hospital model with characteristics of the Enlightenment had never been described and brings new values to those already known in the oldest hospital in Spain still in operation. tration.

Keywords: Sebastián Van der Borcht; hospital architecture; leprosy; military engineers; Illus- 
Sebastián Van der Borcht y Pangaert es sin duda uno de los máximos representantes de la arquitectura sevillana del siglo XVIII. Esto sería así incluso si su único trabajo en Sevilla fuera el que realizó en la Fábrica de Tabacos, edificio en el que su labor es unánimemente reconocida como determinante ${ }^{1}$, tanto en la conformación del proyecto como en la ejecución de las obras. Sabemos que se hizo cargo de estos trabajos por Real Orden del 9 de agosto de 1750. Su trabajo fue altamente considerado por los reyes Fernando VI y Carlos III, y por su ministro Esquilache, que le recompensó con su ascenso hasta el grado de teniente coronel $^{2}$. A pesar de esto fue abruptamente cesado el 1 de julio de $1766^{3}$, tras la caída en desgracia de Esquilache, y destinado al Puerto de Santa María ${ }^{4}$. Durante los dieciséis años que este ingeniero militar, natural de Bruselas ${ }^{5}$, estuvo destinado en Sevilla, realizó otros trabajos en importantes edificios de la ciudad ${ }^{6}$. Entre 1754 y 1756, intervino en la Capilla Real ${ }^{7}$ de la catedral de Sevilla y, a partir de 1757, se le encomendaron varias obras en los Reales Alcázares, donde las reparaciones eran muy numerosas debido a los daños que el edificio sufrió a consecuencia del terremoto de Lisboa en $1755^{\circ}$. De su trabajo en los Reales Alcázares se conservan varios planos (Figura 1), que nos confirman la opinión expresada por Cuevas

${ }^{1}$ Sobre la Fábrica de Tabacos de Sevilla y la importancia de la participación de Van der Borcht es posible consultar: CUEVAS ALCOBER, Luis: Un ejemplar español de arquitectura industrial del siglo XVIII. Sevilla, 1946; SANCHO CORBACHO, Antonio: Arquitectura Barroca Sevillana del siglo XVIII. Sevilla, 1952; y MORALES SÁNCHEZ, José: La Real Fábrica de Tabacos. Arquitectura territorio y ciudad en la Sevilla del siglo XVIII. Sevilla, 1991.

2 SANCHO CORBACHO, Antonio: Arquitectura Barroca ..., op. cit., p. 346.

${ }^{3}$ CUEVAS ALCOBER, Luis: Un ejemplar español..., op. cit., pp. 22-23.

${ }^{4}$ Ibidem, p. 44.

${ }^{5}$ La cuestión del origen de Van der Borcht quedó resuelta por FERNÁNDEZ CACHO, Yolanda: "Documentos de interés biográfico en la investigación artística: Disposiciones de última voluntad en la Sevilla del Siglo XVIII. El testamento de Sebastián van de Borcht", Atrio, 4, 1992, p. 85.

${ }^{6}$ Un sintético pero interesante resumen, con algunas ausencias, de las obras de Van der Borcht en la ciudad en AGUDELO HERRERO, Joaquín: "La obra arquitectónica del ingeniero militar Sebastián van der Borcht", en Arquitectura e iconografía artística militar en España y América (siglos XV-XVIII): actas de las III Jornadas Nacionales de Historia Militar. Sevilla, 1993, pp. 313-328.

${ }^{7}$ MORALES, Alfredo J.: La Capilla Real de Sevilla. Sevilla, 1979. Sobre la intervención de Van der Borcht ver GÁMEZ CASADO, Manuel. "El ingeniero Sebastián van der Borcht en la Capilla Real de Sevilla", en Las artes de un espacio y un tiempo: el setecientos borbónico. Sevilla, 2015, pp. 325-343.

${ }^{8}$ Sobre la intervención de Van der Borcht en los Reales Alcázares ver GESTOSO PÉREZ, José: Sevilla monumental y artística. T. I. Sevilla, 1889, p. 673; y, más recientemente, OLLERO LOBATO, Francisco: "La reforma del palacio gótico de los Reales Alcázares de Sevilla en el siglo XVIII”, Laboratorio de Arte, 11, 1998, pp. 233-252. 
Alcober de que debía ser buen dibujante, motivo por el que, según nos indica, fue destinado el mismo año en que comenzó a prestar servicio en Andalucía, 1745, a formar parte del séquito del ingeniero general ${ }^{9}$. El último trabajo de importancia que hasta el momento se le atribuye es la dirección de las obras de reforma en la Casa de la Moneda, que comenzaron en 1761 y finalizaron en $1763^{10}$.

Además de los proyectos y obras citados, también conocemos dos informes realizados por Van der Borcht. El primero de ellos fue un reconocimiento de la Torre del Oro en septiembre de 1758, antes de que comenzasen las obras de restauración de la misma, que también eran consecuencia de los daños que sufrió en el terremoto de $1755^{11}$. El segundo informe le fue encargado, probablemente en 1760, por el director de la Fábrica de Artillería, Francisco Molina sobre el proyecto y obra que había ejecutado su antecesor en el cargo, Juan Manuel Torres ${ }^{12}$.

Como acabamos de ver, este ingeniero militar tuvo una prolífica actividad durante los años que residió en Sevilla, trabajando siempre en edificios vinculados con la Corona, lo cual es lógico, si pensamos que los ingenieros militares eran un cuerpo al servicio del estado, cuyos miembros estaban perfectamente capacitados para ocuparse de la construcción y el mantenimiento de edificios ${ }^{13}$. En sus estudios, que por entonces se cursaban en la Real Academia Militar de Matemáticas y Fortificación de Barcelona, recibían formación en materias como Obras Públicas o Arquitectura Civil, incluyendo esta última los órdenes arquitectónicos, la proporción y la simetría de las diferentes partes del edificio ${ }^{14}$. Entendemos, por tanto, que era obligado para el rey acudir a ellos para solicitar sus servicios en cualquier problema que tuviera en los edificios de su propiedad.

El edificio que tratamos también era propiedad de la Corona, ya que el Real Hospital de San Lázaro de Sevilla, que mantuvo el patronazgo real hasta 1854,

${ }^{9}$ CUEVAS ALCOBER, Luis: Un ejemplar español..., op. cit., p. 43.

${ }^{10}$ Sobre esta intervención de Van der Borcht ver ESPIAU EIZAGUIRRE, Mercedes: La Casa de la Moneda de Sevilla y su entorno: historia y morfología. Sevilla, 1991.

${ }^{11}$ Según AGUDELO HERRERO, Joaquín: "La obra arquitectónica...”, op. cit., p. 327 , este reconocimiento es el origen de la atribución a Van der Borcht de las obras en la Torre del Oro.

${ }^{12}$ MORA PIRIS, Pedro: La Real Fundición de Bronces de Sevilla. Siglos XVI a XVIII. Sevilla, 1994, pp. 85-87. El autor no indica la fecha de este informe, pero a través de Enrique de la Vega, que incluye en su obra sobre la Real Fundición un listado cronológico de los directores de la misma, sabemos que Francisco Molina tomó posesión del cargo en noviembre de 1759. DE LA VEGA VIGUERA, Enrique: Sevilla y la Real Fundición de Cañones. Sevilla, 1982.

${ }_{13}$ Tenemos que entender que el significado del término ingeniero en el siglo XVIII era diferente al que tenemos actualmente, especialmente tras los cambios en la sociedad y la tecnología que supuso la Revolución Industrial.

${ }^{14}$ MORA PIRIS, Pedro: La Real Fundición..., op. cit., p. 71. 
fue probablemente fundado por Fernando III tras la reconquista ${ }^{15}$. Tenemos noticia de que se había redactado un proyecto para el mismo hacia 1765, a través de una carta remitida por el médico del hospital al asistente de Sevilla en ese año ${ }^{16}$. Como parte de esta investigación hemos accedido a este documento: Contestacion de la Sociedad sobre el Nuevo plano p el Hospital Real de San Lazaro ${ }^{17}$ (Figura 2), que se conserva en el archivo de la Academia de Medicina de Sevilla y que trascribimos a continuación:

\section{"Señor Asistente}

La Sociedad me manda devolverle como ha reconocido atentamente el plano para el nuevo Hospital de S. Lázaro, que Vs se sirvio dirigirle para su examen y considerado el proyecto y distribuciones, le han parecido muy arregladas a su destino, estando en la segura confianza de que el Autor de la Fabrica, que es miembro de la Sociedad save muy á fondo que las habitaciones aplicadas á los enfermos deben tener toda la amplitud, altura, y ventilación, que exigen sus circunstancias o como el quarto sobre el pileño, trae ventado Tiene que responder la Sociedad, reservando su parecer en lo respectivo a la formalidad del Hospital, para quando lo tenga el honor de ser preguntada sobre ese articulo, que es el mas importante.

Sevilla, y marzo 26 de $1765^{\prime \prime}$

La carta está firmada por Bonifacio Ximénez de Lorite, miembro de la Academia de Medicina, que había sido designado por esta para expresar su opinión sobre el proyecto para el nuevo Hospital Real de San Lázaro. Este médico, nacido probablemente en la segunda década de la centuria ${ }^{18}$, ingresó en la Regia Sociedad de Medicina y otras Ciencias de Sevilla en $1756^{19}$, de la que llegó a ser vicepresidente en $1791^{20}$. Pertenecía también a la Academia de Buenas Letras y estaba vinculado a otras instituciones progresistas e ilustradas.

Fueron numerosas las disertaciones que este intelectual ilustrado presentó en la Regia Sociedad y, como destacado especialista en lepra, no en vano ocupó

${ }^{15}$ Sobre el hospital de San Lázaro ver CÓMEZ RAMOS, Rafael: "El hospital de San Lázaro en Sevilla: de fundación medieval a edificio renacentista", Laboratorio de Arte, 3 , 1990, pp. 33-44.

${ }_{16}$ MORENO TORAL, Esteban: Estudio social y farmacoterapéutico de la lepra. El hospital de San Lázaro de Sevilla (siglos XIII-XIX). Sevilla, 1997, p. 68.

${ }^{17}$ AAMS (Archivo de la Academia de Medicina de Sevilla), leg. 1765, Carta de Bonifacio Ximénez de Lorite al asistente de Sevilla.

${ }^{18}$ CARMONA GARCÍA, Juan Ignacio: "Semblanza y mentalidad de un médico ilustrado sevillano: don Bonifacio Jiménez de Lorite", en Andalucía Moderna: actas del II Congreso de Historia de Andalucía. T. 9 (Historia Moderna III). Córdoba, 1995, p. 96.

19 HeRMOSILla MOLINA, Antonio: Cien años de medicina sevillana. Sevilla, 1970, p. 728.

${ }^{20}$ Ibidem, p. 102. 
el cargo de médico del hospital de San Lázaro durante más de veinte años, pronunció dos discursos en la Academia sobre esta enfermedad. El primero de ellos, Instruccion medico legal sobre la lepra, para servir a los Reales Hospitales de San Lázaro, en 1765, y el segundo, Sobre la lepra. Instrucciones medico-prácticas para los Reales Hospitales de San Lázaro, en 1788. Ambos se publicaron en las Memorias Académicas correspondientes a los años indicados y es posible consultarlos actualmente en el Archivo de la Academia de Medicina. En estas disertaciones insistía, contra la opinión general, en la idea de que la lepra tenía cura, y distinguía entre los enfermos dos tipos bien diferenciados, los incipientes y los confirmados. Los primeros podían ser socorridos con medicinas de la época, baños terapéuticos u otros remedios antes de ser recibidos en el hospital. Por su parte, entre los confirmados había observado curación en ambos sexos, por lo que la enfermedad no era tan incurable como se pensaba y por ello debía intentarse su curación ${ }^{21}$. Este convencimiento de la posibilidad de curación y la distinción entre incipientes y confirmados había llevado a Lorite a solicitar en el año 1765 al asistente de Sevilla que los enfermos en el hospital se dividieran según el grado de su enfermedad: "El año pasado de 65 propuse al difunto Asistente Dn Ramon de Larumbe, qe siendo dignos de curarse esos Leprosos, y no teniendo entrada en ninguno de los hospitales de esta ciudad, era mui propio de su oficio qe se admitieran en el de Sn Lazaro, donde habiendo dos apartamients, uno de curacion y otro de incurables, se repartieran, segun su estado"22.

Este convencimiento del médico de San Lázaro de la posibilidad de curación de los enfermos fue clave en la transformación del hospital. En el siglo XVIII los lazaretos españoles aún mantenían el primitivo esquema de hospital-hospicio, que podríamos asimilar al de un convento, donde los enfermos residían en las "casas de los enfermos", similares a las celdas de un convento, hasta el fin de sus días $^{23}$. Hecho al que sin duda había contribuido el carácter incurable que hasta entonces se le suponía a la lepra. Mientras que desde el siglo XVI otros hospitales habían comenzado su evolución hacia un concepto sanitario, las leproserías aún mantenían el esquema formal del modelo monástico.

De la carta de Ximenez de Lorite podemos extraer varios datos de interés. En primer lugar, el hecho mismo de que exista una carta escrita por un médico dando

21 Tomado de MORENO TORAL, Esteban: Estudio social..., op. cit., p. 153.

22 XIMÉNEZ DE LORITE, Bonifacio: "Sobre la lepra. Instrucciones medico-prácticas para los Reales Hospitales de San lázaro”, en Memorias Académicas de la Regia Sociedad de Medicina y otras Ciencias de Sevilla. Sevilla, 1788, pp. 301-302.

${ }^{23}$ Mientras que en el resto de Europa la incidencia de la lepra había descendido notablemente desde el siglo XV, en España, debido según algunos autores, a la inexistencia de leproserías en los reinos musulmanes, esta enfermedad aún estaba presente en el siglo XVIII, existiendo dos focos endémicos en Levante y Andalucía, siendo además este último el motivo de su difusión en Hispanoamérica. 
su opinión sobre un proyecto de arquitectura, que lo ubica claramente dentro de la arquitectura hospitalaria propia de la Ilustración, ya que es una de las características definitorias del modelo hospitalario ilustrado, que los arquitectos asumieran las reflexiones de los médicos, y se subordinaran al criterio de los mismos ${ }^{24}$.

En la carta se refiere a "el plano para el nuevo Hospital Real de S. Lázaro", lo que indica que se trata de una actuación importante, de la que se mencionan características como altura, ventilación, etc., es decir, elementos relacionados con los nuevos criterios de higiene y salubridad que también aportó el concepto ilustrado de la arquitectura y el urbanismo. Más adelante se indica que el autor del proyecto es "miembro de la Sociedad". La Regia Sociedad de Medicina y otras Ciencias tenía entre sus socios miembros de diferentes disciplinas científicas, pero nos pareció extraño desde que accedimos a la carta que formara parte de esta sociedad, eminentemente médica, un profesional con capacidad técnica para realizar un proyecto arquitectónico. Afortunadamente, la obra Cien años de medicina sevillana incluye el listado de los miembros de la sociedad en el siglo XVIII, indicando su fecha de ingreso. Entre los 417 miembros de la sociedad registrados en el siglo XVIII, la mayoría son, evidentemente, médicos o cirujanos, el segundo grupo más numeroso es el de los miembros del clero, le siguen los boticarios, un buen número de abogados y una amplia variedad de otros titulados como botánicos, físicos, matemáticos, etc., pero no aparece ningún arquitecto, y tan solo un ingeniero militar: "24-1-1760, Van der Borcht, Sebastián: Coronel Ing. en la obra de la Real Fábrica de Tabacos de Sevilla. Académico de Buenas Letras ${ }^{25}$. Socio Supernumerario" 26 .

Esta relación de los ingenieros militares con la arquitectura hospitalaria es característica del modelo de hospital ilustrado en el que la importante labor ejercida por estos es conocida y ha sido estudiada por varios autores, como la citada Fernández Mérida, desde una perspectiva amplia de la evolución de las tipologías hospitalarias ${ }^{27}$, más específicamente por Riera, que define al Cuerpo de Ingenieros como "auténtico motor de la tecnología y construcción española de la Ilustración"28, o por Muñoz Corbalán, que los relaciona con la política sanitaria y hospitalaria de la corona borbónica ${ }^{29}$.

${ }^{24}$ FERNÁNDEZ MÉRIDA, María Dolores: "Aproximación a la historia de la arquitectura hospitalaria”, Cuadernos de arte e iconografia, 29, 2006, p. 59.

${ }^{25}$ También en esta Academia eran compañeros Van der Borcht y Ximenez de Lorite.

${ }^{26}$ HERMOSILLA MOLINA, Antonio: Cien años..., op. cit., p. 727.

${ }^{27}$ FERNÁNDEZ MÉRIDA, María Dolores: "Aproximación a la historia...”, op. cit., p. 58.

${ }_{28}$ RIERA, Juan: "Organización hospitalaria militar en la España Ilustrada (Las Ordenanzas de 1739)”, Asclepio, XXVI-XXVII, 1974-1975, pp. 115-134.

${ }^{29}$ MUÑOZ CORBALÁN, Juan Miguel: "Sanidad, higiene y arquitectura en el s. XVIII. Los ingenieros militares: un eslabón en la política sanitaria y hospitalaria 
En la carta también se menciona un plano, que según la misma se había devuelto al asistente de Sevilla. Se ha buscado por tanto este documento en el Archivo Municipal de Sevilla, donde no ha sido posible localizarlo. Sí hemos localizado, en otro archivo de la ciudad, un plano del hospital de San Lázaro, que podemos relacionar con el citado proyecto. Se trata de un dibujo sobre papel de 55,5 x 84,0 cm (Figura 3) que se encuentra en el Archivo Intermedio Militar Sur ${ }^{30}$. La calidad y precisión geométrica de este plano, que es más que aceptable incluso desde parámetros actuales, lo convierten en una herramienta muy valiosa para el estudio de la evolución formal del edificio. Dibujada bajo la planta del edificio, podemos encontrar una escala gráfica en varas castellanas y, en la esquina superior izquierda, una amplia leyenda, denominada: "Esplicacion del Plano del Hospital de San Lázaro", donde se indica el uso de todas las estancias, entre las que podemos encontrar con el número 34 los "quartos de enfermos".

En el momento de su hallazgo nos resultó curiosa su presencia en un archivo militar, pero la relación del coronel Van der Borcht con la Academia de Medicina, que descubrimos más tarde, dio sentido a esta circunstancia, que finalmente apoya nuestra hipótesis ${ }^{31}$. También es posible argumentar la autoría de este plano por parte del ingeniero militar, si lo comparamos con los que dibujó de los Reales Alcázares (Figura 1), con los que guarda grandes similitudes.

En nuestra opinión el plano de San Lázaro es un levantamiento, es decir, un documento redactado como parte de la necesaria toma de datos previa a la elaboración de un proyecto, por lo que lo datamos entre 1760, fecha del ingreso de Van der Borcht en la Academia, y 1765, fecha en la que ya estaba redactado el plano de proyecto, al que se refiere Lorite en su carta. El levantamiento previo es un plano que no suele entregarse al cliente -más interesado en el nuevo proyecto-, motivo por el que permaneció entre los papeles propios de Van der Borcht y finalmente depositado en el archivo militar.

En la carta de 1765 se indica que se ha "considerado el proyecto y distribuciones", es decir, se trata de obras que aún no se han realizado en esa fecha. Pero a tenor de la segunda de las disertaciones de Ximénez de Lorite, leída en 1788, y

borbónica”, Boletín de Arte, 11, 1990, p. 128.

${ }^{30}$ No está firmado ni fechado, pero podríamos datarlo, por analogía con planimetría de la época, en el siglo XVIII. Más adelante, como parte de este artículo, plantearemos una posible fecha para su ejecución más precisa.

${ }_{31} \mathrm{Si}$ bien este plano no es inédito, ya que una pequeña reproducción del mismo apareció en la página 283 del Diccionario Histórico de las calles de Sevilla, dado el ámbito más generalista del mismo y la calidad gráfica con la que se incluyó en esta obra, este plano no había sido analizado por ninguna de las publicaciones previas sobre el hospital de San Lázaro. Por otro lado, debido probablemente a un fallo de imprenta, la referencia bibliográfica del plano estaba erróneamente indicada en dicha obra, pero gracias a la inestimable colaboración de la Directora Técnica del Archivo Intermedio Militar Sur, Rocío de los Reyes Ramírez, ha sido posible recuperar este interesante documento. 
en la que podemos leer "En efecto se tomaron las correspondientes medidas, y se elaboraron los planos, q precisamente se hallaron en el archivo de la Intend ${ }^{\text {a }}{ }^{32}$, entendemos que sus recomendaciones fueron tenidas en cuenta.

Gracias a los datos expuestos mantenemos la hipótesis de que el proyecto para el nuevo hospital de San Lázaro fue redactado por Sebastián Van der Borcht, aunque no creemos que le fuera posible ejecutar las obras del mismo. En primer lugar, por la cercanía entre la fecha de elaboración del proyecto, 1765, y la de su traslado al Puerto de Santa María en 1766. En cualquier caso, gracias a varios documentos localizados en el Archivo de la Diputación Provincial de Sevilla, podemos suponer que finalmente se ejecutaron las obras proyectadas, y confirmar los cambios que estas obras supusieron para el tratamiento de los enfermos.

El primero de estos documentos es el Inventario de los muebles alajas y efectos del Real Hospital de San Lázaro de Sevilla, realizado en $1812^{33}$. Este inventario trata sobre bienes muebles, pero estos se enumeran indicando en qué dependencia se encuentran, mencionando, por tanto, las estancias con las que entonces contaba el edificio, que eran las siguientes: "Iglesia (Nave del norte y nave del mediodia); Sacristia; Quarto de cemento; Quarto del Cura, Claveria, Enfermeria alta ${ }^{\mathrm{a}}$ hombres, Enfermeria alta de mujeres, Granero alto, Crujia de la calle (Casa alta del Sr Mayoral y un postigo pal.); Enfermeria baja de hombres; mujeres y roperia; Refectorio, Cocina; Despensa; Almacen de Aseyte; Lavaderos; Quarto de prisiones; Guardilla; Cavalleriza; Carbonera y leñeria; Porteria".

Lo más significativo de este listado es que ya no se mencionan, como había sido habitual hasta entonces, las casas de los enfermos, y que aún podíamos ver dibujadas en el levantamiento que atribuimos a Van der Borcht, en el que las denomina "quartos". Por el contrario, en el inventario de 1812, se describen enfermerías diferenciadas por sexo y estadio de la enfermedad. Según este documento, algunos elementos de las enfermerías aún permanecían sin acabar, pero tanto en la "Enfermeria alta $\mathrm{p}^{\mathrm{a}}$ hombres" como en la "Enfermeria alta de mujeres" se había dispuesto una pila. Esto nos hace suponer que las enfermerías altas, en las que los enfermos compartían un solo espacio o cuadra ${ }^{34}$, estaban destinadas a los enfermos incipientes, con posibilidad de curación, ya que parte de los tratamientos de la época estaban basados en baños curativos ${ }^{35}$. Por otro lado, "la enfermeria baja de hombres y mujeres" sería probablemente la que estaba destinada a los incurables, y por esta razón aún conservaba reminiscencias de la vieja estructura, y

32 XIMÉNEZ DE LORITE, Bonifacio: “Sobre la lepra...”, op. cit., p. 302.

33 ADPS (Archivo de la Diputación Provincial de Sevilla), HSL (Hospital de San Lázaro), leg. 25.

34 ADPS, HSL, leg. 25, Inventario de los muebles, alajas y efectos del Real Hospital de San Lázaro de Sevilla, pp. 11-12.

${ }^{35}$ MORENO TORAL, Esteban: Estudio social..., op. cit., p. 153. 
todavía se mencionan en ella "quartos de los pobres"36, probablemente habitaciones independientes, agrupadas en la enfermería de planta baja.

En 1814 se redactó un segundo documento que fue titulado por su autor, don José María Murta Donaire y Figueroa, capellán del hospital: Noticia Razonada sobre el Real Hospital de San Lázaro año de 1814 ${ }^{37}$. De la lectura de este informe se deduce aún más claramente que la intención de separar a los enfermos, entre curables e incurables, era ya un hecho y en esa fecha se diferenciaban y trataban a los leprosos conforme a las recomendaciones de Ximénez de Lorite: "que se revisen los malatos, elefantiasos o leprosos en cualquier grado: los uno para que con la correspondiente separacion se los tratare de cuidar, pues no hay hospitalidad alguna con ese destino: y los otros ó los incurables para que recivieren, como en efecto reciben los oficios de humanidad y beneficiencia que inspira naturaleza" 38 .

Es decir, tenemos constancia de que al menos desde 1812, el hospital reflejaba un cambio sustancial en su formalización y funcionamiento; los enfermos ya no residían en casas o "quartos" sino en enfermerías comunes. Es importante resaltar que se trata de la primera vez que podemos leer la palabra enfermería en una descripción del hospital. Este cambio supuso mucho más que una modificación de su distribución formal. Nos indica que se había fraguado un nuevo concepto de la enfermedad y de su tratamiento.

Tras la reforma de finales del siglo XVIII, el hospital había comenzado el siglo XIX evolucionando hacia lo que actualmente entendemos por un establecimiento sanitario, pero esta tendencia se detuvo bruscamente, e incluso sufrió un retroceso debido al levantamiento de 1820 y el posterior Trienio Liberal (18201823). Este periodo supuso para San Lázaro la pérdida de sus privilegios reales ${ }^{39}$, lo que fue especialmente perjudicial en lo relativo a los ingresos del hospital. A través del documento Visita de médicos al hospital de San Lázaro el 8 de Marzo de $1821^{40}$, sabemos que la situación del establecimiento era tan precaria un año después que tan solo malvivían en él diez pacientes de los considerados incurables, y los enfermos considerados curables eran devueltos a sus casas ${ }^{41}$. En estos tres años, el establecimiento incluso dejó de prestar cualquier asistencia durante periodos

${ }^{36}$ ADPS, HSL, leg. 25, Inventario de los muebles, alajas y efectos del Real Hospital de San Lázaro de Sevilla, p. 12.

${ }^{37}$ ADPS, HSL, leg. 4. Este documento no ha sido considerado, hasta esta investigación, en ninguno de los estudios sobre la evolución arquitectónica del hospital de San Lázaro.

${ }^{38}$ ADPS, HSL, leg. 4, Noticia Razonada sobre el Real Hospital de San Lázaro año de 1814 , p. 16.

39 GIMÉNEZ MUÑOZ, María del Carmen: Los establecimientos benéficos más relevantes de Sevilla hasta 1849. Sevilla, 2008, p. 76.

40 ADPS, HSL, leg. 51.

${ }^{41}$ ADPS, HSL, Visita de médicos al hospital de San Lázaro el 8 de Marzo de 1821, pp. $15-16$. 
temporales "por la absoluta carencia de fondos para sufragarlo"42. Finalmente, esta dura etapa se solventó con la supervivencia del hospital, pero supuso un retroceso del lazareto hacia el modelo anterior que ya había sido superado gracias a su reforma ilustrada.

Este abandono de las enfermerías diferenciadas según el estadio de la enfermedad se puede comprobar en los siguientes documentos que hemos localizado. El primero de ellos es el Memorial Histórico-Descriptivo del Real Hospital de san Lázaro de Sevilla, redactado por el doctor en medicina y cirugía Nicolás Moreno en 1829, que se conserva en la Real Academia de Medicina de Cádiz, en el que tan solo indica la separación de enfermos en "dos departamentos uno de hombres y de mujeres el otro" ${ }^{43}$, y al parecer accedió al destinado a los varones ya que describe en él "once habitaciones señaladas para los enfermos" 44 .También nos informa de este abandono de la enfermerías la Noticia artística, histórica y curiosa... que publicó en 1844 Félix González de León, obra en la que se realiza una somera descripción del hospital, en la que cuando se refiere a la parte destinada para habitar los enfermos indica "que viven en cuartos separados" 4 .

Como acabamos de ver en los documentos de la década de 1810, se seguía hablando de enfermerías, distribuidas conforme al proyecto redactado por Van der Borcht, según el modelo propuesto por Ximénez de Lorite: enfermerías altas para enfermos tratables y enfermerías bajas, divididas en "quartos de los pobres" para los incurables. Sin embargo, no se mencionan en los textos posteriores a 1820. Ya fuera porque las plantas altas, faltas de mantenimiento, se degradaron hasta el punto de hacerlas inhabitables, o porque los enfermos abandonados a su suerte se trasladaron a las habitaciones individuales de la planta baja, el hecho cierto es que desaparecieron tanto las enfermerías como los tratamientos que se prestaban a los enfermos con posibilidad de curación.

Será necesario esperar hasta otra obra ejecutada en el hospital entre 1860 y 1864 para que se vuelvan a mencionar enfermerías en San Lázaro, pero al mismo tiempo esta intervención y, más tarde, las numerosas obras llevadas a cabo durante el siglo XX transformaron de tal manera el edificio que, hoy en día, es muy difícil determinar cómo se habían detallado las obras de la segunda mitad del siglo XVIII.

${ }^{42}$ CUENCA TORIBIO, José Manuel: Historia de Sevilla. Del Antiguo al Nuevo Régimen. Sevilla, 1976, p. 70.

${ }^{43}$ RUIZ VEGA, Paloma: "El Hospital de San Lázaro de Sevilla, según documentación de la Real Academia de Medicina de Cádiz en 1829", en Memorias Académicas de la Real Academia de Medicina y Cirugía de Sevilla. Sevilla, 2014, p. 109.

44 Ibidem.

${ }^{45}$ GONZÁLEZ DE LEÓN, Félix: Noticia artística, histórica y curiosa de todos los edificios públicos, sagrados y profanos de esta Muy Noble, Muy Leal, Muy Heroica e Invicta Ciudad de Sevilla. T. II. Sevilla, 1844, pp. 247-248. 
Sí que podemos, gracias a un segundo plano localizado en el archivo de la Diputación de Sevilla, entender de manera general cómo modificó el proyecto del siglo XVIII la estructura general del hospital ${ }^{46}$. Se trata de un dibujo sobre papel de 65 x $93 \mathrm{~cm}$ (Figura 4), firmado por Manuel de la Vega y Antonio de Padura en junio de 1890, por lo que es posible observar el resultado de las obras de 1860-1864. Esta intervención del siglo XIX, que se denominaba en el expediente administrativo "obra general de reparación importante", se ejecutó conforme al proyecto redactado por Balbino Marrón ${ }^{47}$, que también se conserva en la Diputación de Sevilla y que fue titulado por su autor Proyecto de reparación del Hospital de San Lázaro. De la lectura del mismo podemos extraer varios datos que nos ayudan a entender la intervención ejecutada en el siglo XVIII. En primer lugar, el autor de la memoria indica que desde principios del siglo XIX no se habían ejecutado obras en el hospital ${ }^{48}$. Por otro lado, como indican el título del expediente y el del proyecto, y como nos revela una lectura detallada del mismo ${ }^{49}$, la intervención de Balbino Marrón fue una rehabilitación que afectó principalmente a la crujía de fachada, de la que tan solo alteró su distribución interior ${ }^{50}$.

Si estas obras fueron las primeras del siglo XIX, y no alteraron la estructura general del edificio, podemos suponer que el esquema funcional del hospital que se observa en el plano de 1890, en el que el edificio se desarrolla en torno a dos patios, uno de entrada y otro destinado a las enfermerías, a la izquierda, existía antes de la intervención de Balbino Marrón. Entendemos, por tanto, que la distribución en torno a dos patios era el resultado de la intervención de finales del siglo XVIII. Esta es diferente a la que se puede observar en el plano que hemos datado en la primera mitad de la década de 1760, en el que el hospital aún se dibujó organizado en torno a tres patios, el de entrada y, a la izquierda de este, otros dos patios donde se encontraban los "quartos de enfermos".

El análisis comparado de los textos de principios del siglo XIX, del plano que hemos datado en 1760-1765 y del plano de 1890 nos permite conocer, por

${ }^{46}$ Localizado y catalogado en el Archivo de la Diputación Provincial de Sevilla en el transcurso de esta investigación. Agradecemos la colaboración del Área de Cultura y Ciudadanía y especialmente de la jefa del Servicio de Archivo y Publicaciones de la Diputación de Sevilla, doña Carmen Barriga Guillén.

${ }^{47}$ VILAPLANA VILLAJOS, Fernando: "Datación del supuesto patio mudéjar del hospital de San Lázaro de Sevilla. Ejemplo del aporte de la disciplina arquitectónica en un estudio patrimonial interdisciplinar", PH Investigación, 2, 2014, p. 83.

48 ADPS, Juntas de Beneficencia, leg. 56, Proyecto de reparación del Hospital de San Lázaro, p. 1.

${ }^{49}$ Para un análisis detallado de esta intervención ver VILAPLANA VILLAJOS, Fernando: El hospital de San Lázaro en Sevilla. Origen y transformaciones. Tesis doctoral inédita. Universidad de Sevilla, 2017.

${ }^{50}$ ADPS, Juntas de Beneficencia, leg. 56, Proyecto de reparación del Hospital de San Lázaro, p. 3. 
tanto, la principal modificación que supuso el proyecto impulsado por Bonifacio Ximénez de Lorite y cuya redacción hemos atribuido a Sebastián van der Borcht. Tras la ejecución de ese proyecto, la zona del hospital destinada a los enfermos se agrupó en torno a un solo patio destinado a albergar las enfermerías. Esta intervención, como se ha dicho, respondió a las características de la arquitectura hospitalaria ilustrada, habiendo contado de manera definitoria para su diseño con las indicaciones de un médico, lo que sin duda influyó en la aplicación de los nuevos criterios sanitarios e higiénicos. Desafortunadamente, la dura etapa que supuso para San Lázaro la década de los años veinte del siglo XIX supuso el olvido de la interesante reforma dieciochesca del hospital. Intervención que hemos intentado esclarecer, a través de la documentación consultada en diferentes archivos.

Esperamos que esta investigación colabore al mayor conocimiento de este hospital que en los últimos años está recuperando el lugar que le pertenece como significativo elemento del patrimonio sevillano, por la gran variedad de valores que atesora. A los que podemos ahora incorporar el de ser uno de los primeros ejemplos que conocemos en nuestra ciudad de la arquitectura hospitalaria de la Edad Moderna, en la que los arquitectos comenzaron a asumir las reflexiones de los médicos. Esto propició que, desde su origen como lugares de asilo, los hospitales dieran un paso más en su evolución para convertirse en centros de curación. Transformación que, como hemos visto, se produjo en el hospital de San Lázaro a finales del siglo XVIII.

Fecha de recepción: 2 de agosto de 2018

Fecha de aceptación: 1 de julio de 2019 


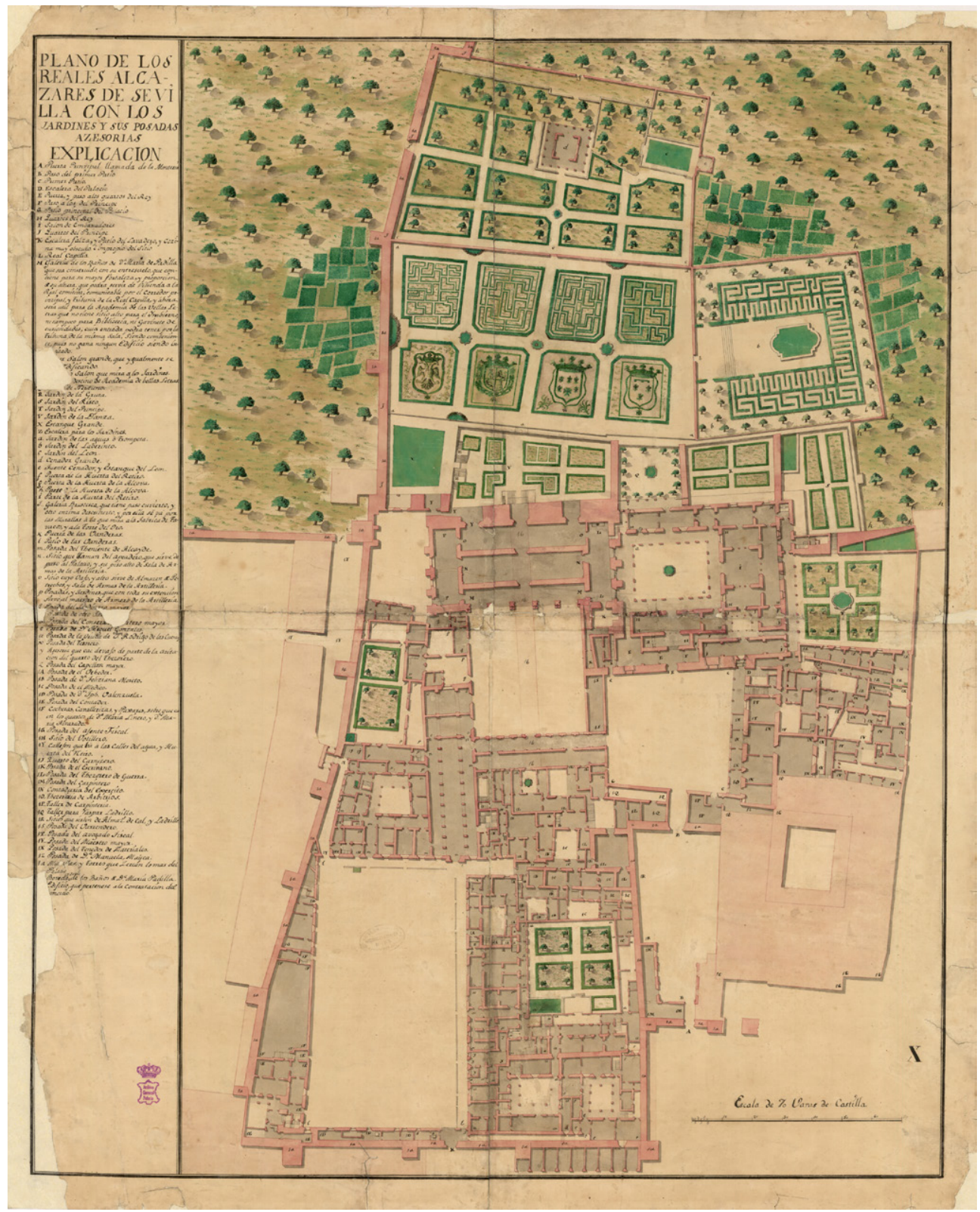

Figura 1. Plano de los Reales Alcázares de Sevilla, Archivo General de Palacio, Planos Mapas y Dibujos, $n^{\circ} 5956$. 


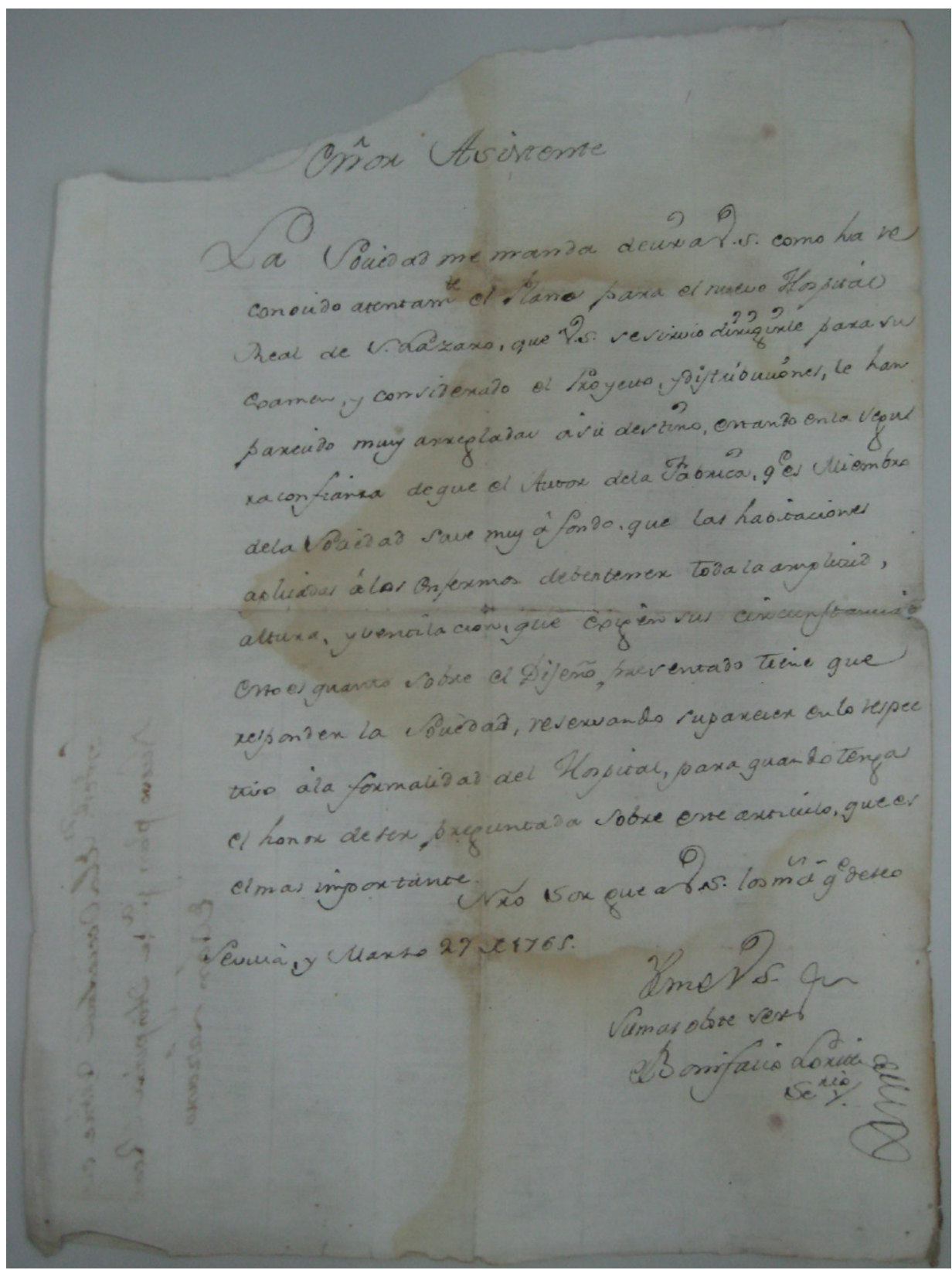

Figura 2. Contestacion de la Sociedad sobre el Nuevo plano pa el Hospital Real de San Lazaro, Archivo de la Academia de Medicina de Sevilla, leg. 1765.

Foto: Fernando Vilaplana Villajos.

LABORATORIO DE ARTE 31 (2019), pp. 387-402, ISSN 1130-5762

e-ISSN 2253-8305 - DOI http://dx.doi.org/10.12795/LA.2019.i31.22 


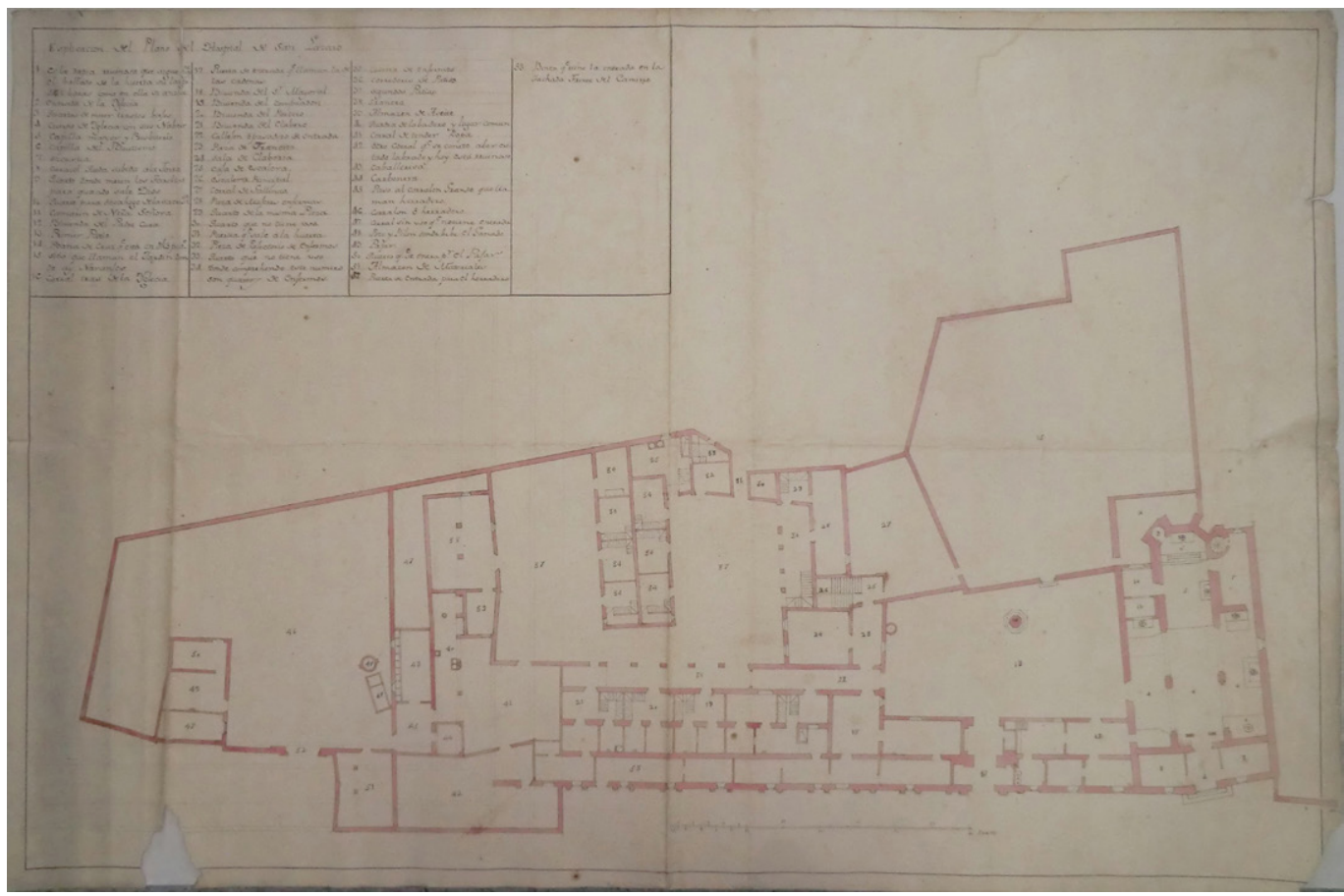

Figura 3. Planta del hospital de San Lázaro, Archivo Intermedio Militar Sur, cajón 8, plano 24. Foto: Fernando Vilaplana Villajos. 


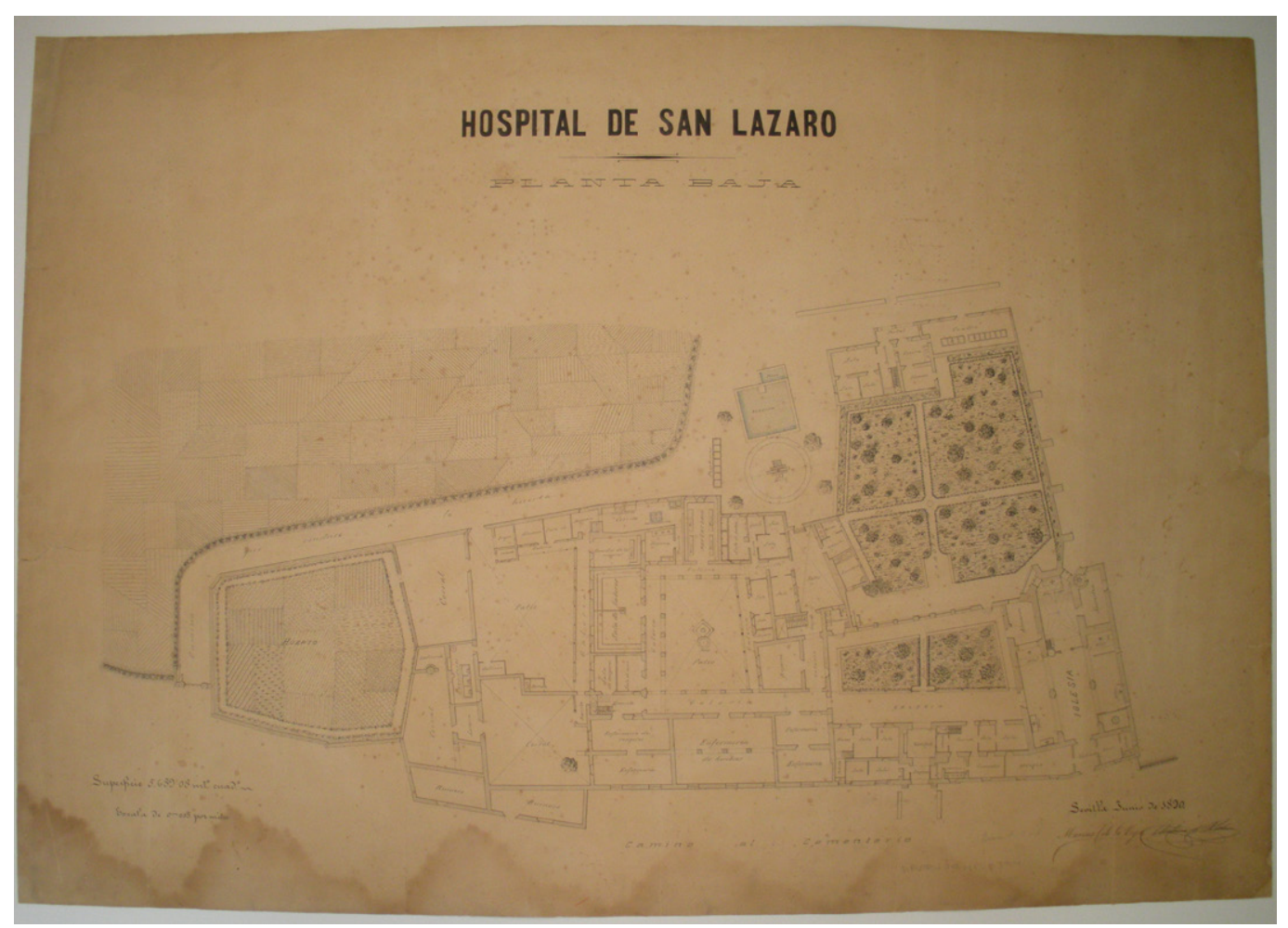

Figura 4. Planta baja del hospital de San Lázaro, Archivo de la Diputación Provincial de Sevilla, Mapas, Planos y Dibujos, 394. 